\section{Maternal Death Due to Sagittal Sinus Thrombosis- Autopsy Diagnosis: A Case Report}

\section{Emre Canverenler ${ }^{1}$, Burcu Kasap $^{2 \star}$, Melike Erbaş ${ }^{3}$, Kenan Karbeyaz ${ }^{4}$, Yasemin Balcl ${ }^{4,5}$ and Eren Akbaba ${ }^{2}$}

'Department of Obstetrics and Gynecology, Sinop State Hospital, Sinop, Turkey

${ }^{2}$ Department of Obstetrics and Gynecology, School of Medicine, Muğla Sıtkı Koçman University, Menteşe, Muğla, Turkey

3Justice Ministry, Institute of Forensic Medicine, Muğla, Turkey

${ }^{4}$ Justice Ministry, Institute of Forensic Medicine, Eskişehir, Turkey

${ }^{5}$ Department of Forensic Medicine, School of Medicine, Muğla Sıtkı Koçman University, Menteşe, Muğla, Turkey

\begin{abstract}
We report a case of 30 years old maternal death due to sagittal sinus thrombosis after delivery via cesarean section. The woman had two previous cesarean deliveries and admitted to emergency department with severe headache and loss of sensation at one side of her leg on the postpartum eighth day. Sagittal sinus thrombosis and intracerebral hematoma were diagnosed by both clinical and radiological examinations. The patient was unable to survive despite the intensive treatment period. Herein, we report the evaluation of autopsy findings by forensic medicine. In conclusion, the evaluation of maternal mortality cases also with autopsy findings might yield accompanying effective factors and might cause a decrease in maternal mortality rates.
\end{abstract}

Keywords: Autopsy; Maternal death; Sagittal sinus thrombosis

\section{Introduction}

Cerebral Venous Thrombosis (CVT) occurs due to occlusion of cerebral venous sinus or cortical veins. The most common form is the Superior Sagittal Sinus (SSS) thrombosis. The coexisting etiologic factors like infection, head trauma, surgical interventions and tumors increase the frequency of thrombosis formation [1]. CVT may be seen in all ages of men and women but incidence rate of CVT increases in

*Corresponding author: Burcu Kasap, Department of Obstetrics and Gynecology, School of Medicine, Muğla Sıtkı Koçman University, Menteşe, Muğla 48000, Turkey; Tel: +90 2522114800/5156; E-mail: burcuharmandar@gmail.com; burcuharmandar@mu.edu.tr

Citation: Canverenler E, Kasap B, Erbaş M, Karbeyaz K, Balcı Y, et al. (2015) Maternal Death Due to Sagittal Sinus Thrombosis-Autopsy Diagnosis: A Case Report. J Clin Stud Med Case Rep 2: 012.

Received: January 06, 2015; Accepted: March 18, 2015; Published: April 01, 2015 fertile women due to oral contraceptive use, pregnancy and postpartum period.

Diagnosis can be confirmed by cerebral angiography, computed tomography and magnetic resonance images. Prognosis is variable and sometimes it may regress spontaneously.

CVT mostly occur in puerperal period of the pregnancy. Cortical venous thrombosis which may lead to cerebral infarction or bleeding is also a complication of pregnancy and mostly occur within three weeks after delivery. The most commonly affected region is the SSS. The incidence rate of SSS thrombosis during pregnancy differ according to the developmental level of the country. The incidence of SSS thrombosis is 1 per 250 pregnant women in India meanwhile its incidence is very low in European countries [2]. This condition can be caused to the lack of diagnostic and treatment facilities. Herein, we report a case of 30 years old maternal death due to sagittal sinus thrombosis after delivery via cesarean section and aim to evaluate autopsy findings.

\section{Case Presentation}

A 30 years old pregnant woman delivered at term by emergent Cesarean Section (CS) due to indication of previous cesarean operations in a tertiary referral training and research hospital. CS was performed under spinal anesthesia. The patient was discharged from the hospital at the third postpartum day in a stable condition without any complications due to operation. She was totally asymptomatic by means of neurological sytem at the time of discharge. But she was admitted to emergency department with severe headache, loss of sensation at one side of her leg and coughing on the postpartum eighthday. She was hospitalized at Neurology Department with the prediagnosis of SSS thrombosis. Although the cranial angiography report revealed normal findings, a hematoma of $37 \times 33 \mathrm{~mm}$ size at left parietal lobe and asymmetrical lateral ventricules were detected via cranial tomography. At this stage intervention was not planned by neurosurgery specialists. Patient was given anticoagulant and anti-edematherapy. Afterwards, the second cranial tomography examination reported a secondary hyperdense focus in vertex and a $2 \mathrm{~mm}$ shift to right side. In the follow-up condition of the patient was further aggravated and comatous state occured. At the sixteenth postpartum day "brain death" diagnosis was accepted by a committee including specialists of neurosurgery, anesthesiology and cardiology at twenty first postpartum day.

\section{Autopsy Findings}

\section{External examination}

Incision site due to CS and evidence of medical interventions.

\section{Internal examination}

There was neither epidural nor subdural hematoma detected inside the cranial cavity. The diameters of the sagittal sinus, sinus rectus and right transverse sinus were enlarged upto $1.5 \mathrm{~cm}$ dimensions and coagulum formations were detected in those enlargements (Figure 1). Intracerebral hematoma of $4 \times 4 \times 2 \mathrm{~cm}$ size in left parietal region and minimal bleeding areas in brain stem were 
detected. A 2 x $2 \mathrm{~cm}$ size of intracerebral bleeding, which was being fed by left medial cerebral artery, was seen by autopsy examination (Figure 2). All the arteries of brain were dilated but there was notan evidence of aneurysma. In thoracal examination, bilateral lungs were found to be larger than normal. In serial cuttings intense edema and intense mucous in bronchi were present.

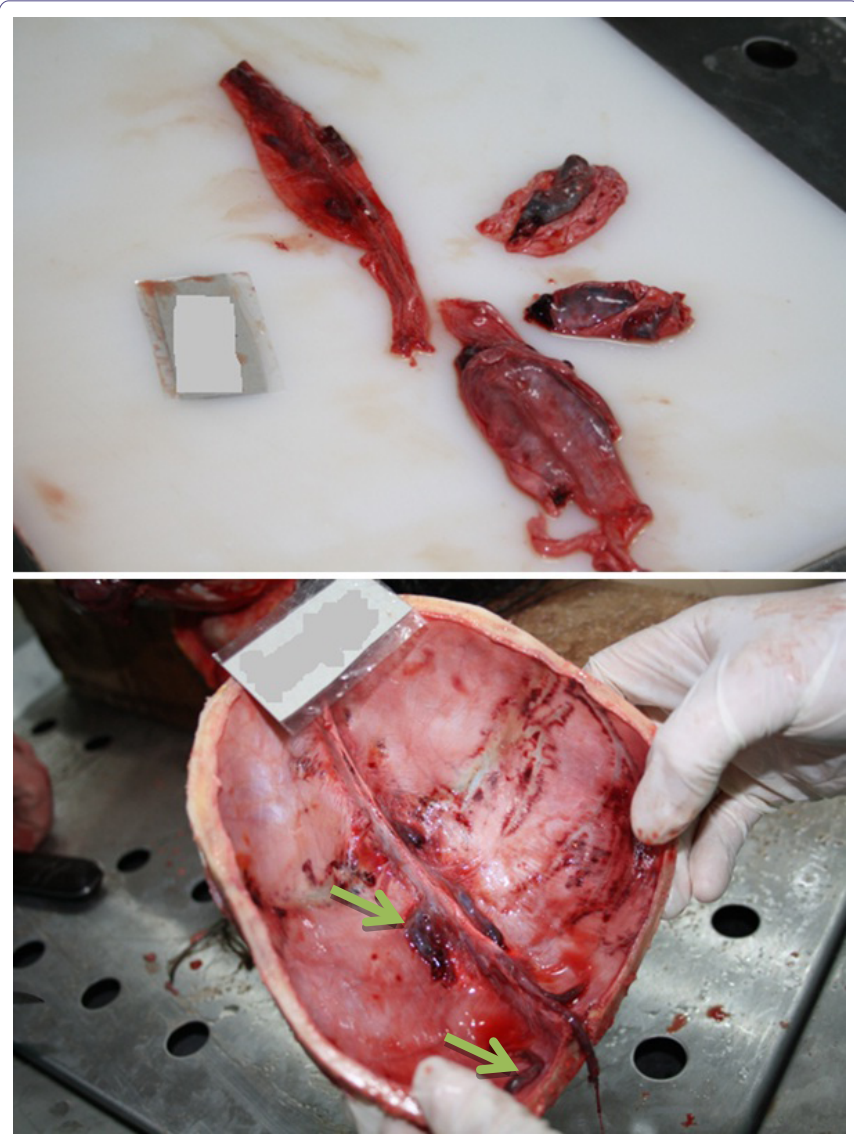

Figure 1: Thrombus Formation in Sagittal Sinus.

\section{Histopathological examination}

Intraparenchymal bleeding points in brain stem, thrombus in sagittal sinus, acute pneumonia, diffuse alveolar destruction and intraalveolar edema were detected histopathologically.

In conclusion, the death of the patient was thought to be caused by pathological bleeding in brain and this etiology was aggravated by severe acute pneumonia.

\section{Discussion}

Thrombosis of cerebral veins or sinuses is a cerebrovascular disease which may be misdiagnosed or diagnosed too late. It may happen in all ages but mostly in young and middle aged women [3]. Clinical presentation may vary and can be so dramatical. It is suddenly presented with headaches due to intracranial hypertension, focal neurological problems, convulsions and lethargy $[3,4]$. The reported case was also presented with headache and lethargy but the situation of the patient got worse during hospitalization. Many etiological factors are reported in the literature but in $20-25 \%$ of the cases exact etiology could not be identified [3]. Mortality rate is 5-30\% [4,5]. Many of the cases might completely recover. But residual neurological sequela in $20 \%$ of the patients and epileptic convulsions in $5 \%$ of the patients may remain [4-6].

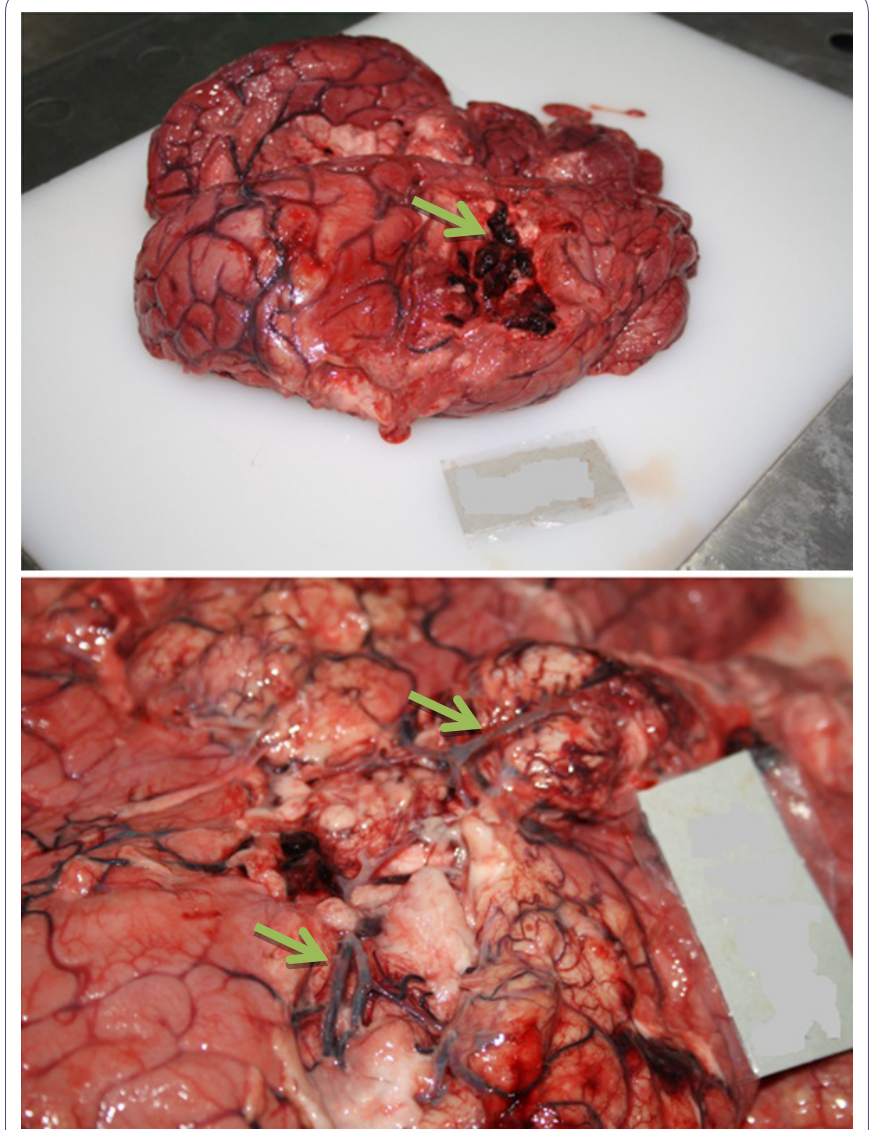

Figure 2: Intracerebral hematoma in the regions being fed by median cerebral artery and dilatation in all cerebral veins.

Severe dehydration, last trimester of the pregnancy, genetical disorders of the coagulation mechanism like protein $\mathrm{C}$ or S deficiency, infections like otitis and mastoiditis or septisemia, malignancy, head trauma and hematological disorders are the major risk factors for CVT [6]. Diagnosis might be performed by advanced neuroradiological examinations [7].

In patients with known genetic predispositions, anticoagulant therapy such as low molecular weight heparin could be done for prevention especially in postpartum period. When SSS thrombosis formed therapy can vary such as corticosteroids, barbiturates, cerebrospinal fluid drainage, craniectomy for decompression, thrombectomy, heparin, local urokinase and tissue plasminogen activators. Although anticoagulant therapy can be successful in some cases, it is still controversial. Some authors have concluded that some patients can heal in three weeks to six months without any medical or surgical treatment.

Pregnancy is a prothrombotic state due to changes in hemostatic sytem. These physiological changes are mandatory for the safety of fetoplacental circulation but it might lead to formation of thrombi. These changes in hemostatis become evident after the second trimester of pregnancy. The levels of fibrinogen, factor VII, VIII, IX, $\mathrm{X}, \mathrm{XII}$, high molecular weight kininogen and prekallikrein increase during pregnanacy $[8,9]$. These changes in hemostatic parameters do not completely return to normal levels in the early postpartum period. So the patients with coexisting etiologic factors for thrombosis may gain extra risk at the postpartum period. In this case report, the clinical symptoms were marked approximately one week after birth 
and with radiological examination the diagnosis of the SSS thrombosis was suspected. An intracerebral hematoma with a size of $3 \mathrm{~cm}$ was detected but notany surgical intervention was thought. Postmortem findings were supportive for the suspected clinical diagnosis.

In this paper, we reported a maternal death by sagittal sinus thrombosis due to predisposition to clotting after birth. The minimal intracerebral hematoma and severe pneumonia were also thought to be effective in this thrombosis predispositon.

\section{References}

1. Iskandar BJ, Kapp JP (1996) Nonseptic venous occlusive disease. In: Wilkins $\mathrm{RH}$, Rengachary SS (eds.). Neurosurgery (2ndedn). McGraw Hill, USA. Pg: 2177-2190.

2. Srinivasan K (1984) Ischemic cerebrovascular disease in the young. Two common causes in India. Stroke 15: 733-735.
3. Kimber J (2002) Cerebral venous sinus thrombosis. QJM 95: 137-142.

4. Agostoni E (2004) Headache in cerebral venous thrombosis. Neurol Sci 25 206-210.

5. Crassard I, Bousser MG (2004) Cerebral venous thrombosis. J Neuroophthalmol 24: 156-163.

6. Bousser MG, Chiras J, Bories J, Castaigne P (1985) Cerebral venous thrombosis--a review of 38 cases. Stroke 16: 199-213.

7. Connor SE, Jarosz JM (2002) Magnetic resonance imaging of cerebral venous sinus thrombosis. Clin Radiol 57: 449-461.

8. McColl MD, Walker ID, Greer IA (1999) The role of inherited thrombophilia in venous thromboembolism associated with pregnancy. Br J Obstet Gynaecol 106: $756-766$

9. Matsuura T, Kobayashi T, Asahina T, Kanayama N, Terao T (2001) Is factor XII deficiency related to recurrent miscarriage? Semin Thromb Hemost 27: $115-120$. 